\title{
THE KAUFFMAN POLYNOMIALS OF GENERALIZED HOPF LINKS
}

\author{
JIANYUAN K. ZHONG AND BIN LU
}

\begin{abstract}
Following the recent work by Chan [4] and Morton and Hadji [7] on the Homflypt polynomials of some generalized Hopf links, we investigate the Kauffman polynomials of generalized Hopf links. By studying the Kauffman skein module of the solid torus $S^{1} \times D^{2}$, we establish a similar skein map on the Kauffman skein module of $S^{1} \times D^{2}$ which has distinct eigenvalues. Furthermore we are able to calculate the Kauffman polynomials of some specific generalized Hopf links.
\end{abstract}

\section{INTRODUCTION}

In [4], Chan discusses the Homflypt polynomials of reversed string parallels $H\left(k_{1}, k_{2} ; n_{1}, n_{2}\right)$ of the Hopf link. Morton and Hadji analyze their structure more closely by using the Homflypt skein of the annulus and identify the eigenvalues and eigenvectors (see [7]). Here we follow a similar approach to Morton and Hadji's to investigate Kauffman polynomials of generalized Hopf links by using the Kauffman skein module of the solid torus (the annulus).

Let $k$ be an integral domain containing the invertible elements $\alpha$ and $s$. We assume that $s-s^{-1}$ is invertible in $k$.

By a framed oriented link we mean a link equipped with a string orientation together with a nonzero normal vector field up to homotopy. By a framed link we mean an unoriented framed link. The links described by figures in this paper will be assigned the vertical framing which points towards the reader.

There are various versions of the Kauffman polynomial in the literature. The version we use in this paper is defined by the following Kauffman skein relations:

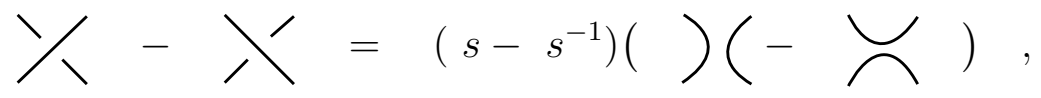

Date: November 28, 2001.

Key words and phrases. Kauffman polynomial, Homflypt skein modules, relative skein modules, Hecke algebra and Birman-Murakami-Wenzl algebra. 


$$
\begin{aligned}
& 1 / \\
& L \sqcup \bigcirc=\alpha /,
\end{aligned}
$$

where $\delta=\left(\frac{\alpha-\alpha^{-1}}{s-s^{-1}}+1\right)$. The last relation follows from the first two when $L$ is nonempty.

Remark. The Kauffman polynomial of the empty link is normalized to be 1 and the Kauffman polynomial of a link $L$ is denoted by $\langle L\rangle$.

Let $M$ be a smooth, compact and oriented 3-manifold.

Definition 1. The Kauffman skein module of $M$, denoted by $K(M)$, is the $k$-module freely generated by isotopy classes of framed links in $M$ including the empty link quotient by the Kauffman skein relations given above.

We consider the unoriented Hopf link with linking number 1 as shown below:

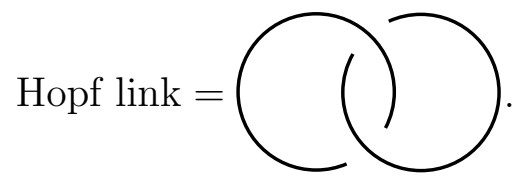

We have that $<$ Hopf link $>=\delta\left[\delta+\left(s-s^{-1}\right)\left(\alpha-\alpha^{-1}\right)\right]$.

A great variety of links can be realized through the construction of satellite links by using the Hopf link [7], which is to take the two components of the Hopf link and decorate them with diagrams in the annulus. If the two components are decorated by $P_{1}$ and $P_{2}$, respectively, we denote the realized satellite link by $H\left(P_{1}, P_{2}\right)$. When $P_{1}$ is $n$ parallel copies of the unknot and $P_{2}$ is $m$ parallel copies of the unknot, we denote $H\left(P_{1}, P_{2}\right)$ by $H(n, m)$. These are the generalized Hopf links. We will label a string with an integer $n$ to indicate $n$ parallel strings.

A picture of $H(n, m)$ is given by the following:

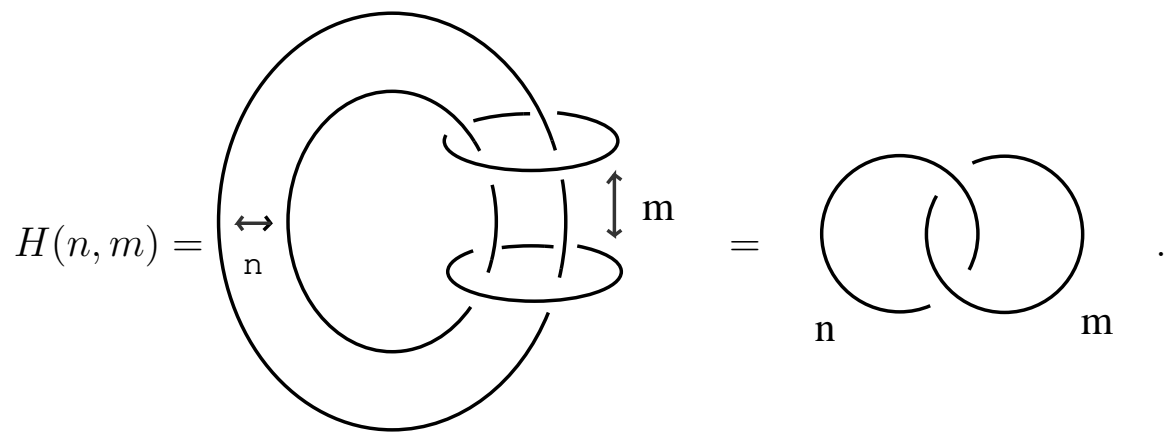

We observe that the links $H(n, m)$ and $H(m, n)$ are equivalent links. 
THE KAUFFMAN POLYNOMIALS OF GENERALIZED HOPF LINKS

2. On the Kauffman Skein module of $S^{1} \times D^{2}$

2.1. Hecke algebras. Here we summarize a geometric realization of the Hecke algebras through the Homflypt skein modules.

Definition 2. The Homflypt skein module of $M$, denoted by $S(M)$, is the $k$-module freely generated by isotopy classes of framed oriented links in $M$ including the empty link, quotient by the Homflypt skein relations given in the following figure.

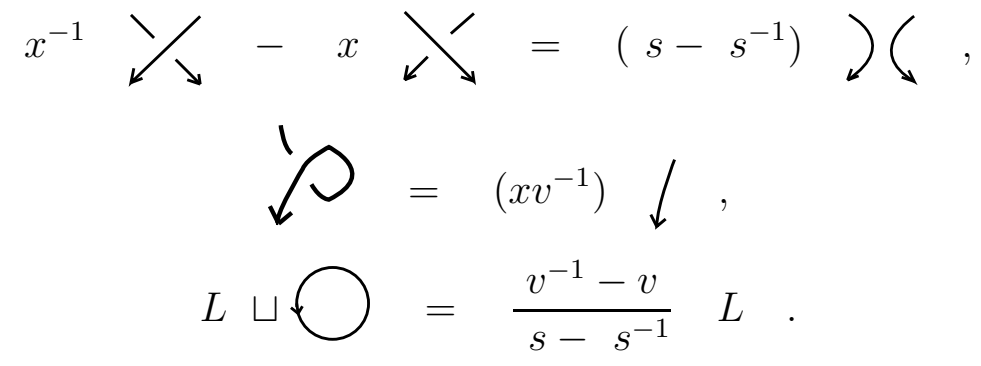

Definition 3. The relative Homflypt skein module. Let $X=$ $\left\{x_{1}, x_{2}, \cdots, x_{n}\right\}$ be a finite set of framed points oriented negatively (called input points) in the boundary $\partial M$, and let $Y=\left\{y_{1}, y_{2}, \cdots, y_{n}\right\}$ be a finite set of framed points oriented positively (called output points) in $\partial M$. The relative skein module $S(M, X, Y)$ is the $k$-module generated by relative framed oriented links in $(M, \partial M)$ such that $L \cap \partial M=$ $\partial L=\left\{x_{i}, y_{i}\right\}$ with the induced framing and orientation, considered up to an ambient isotopy fixing $\partial M$, and quotient by the Homflypt skein relations.

In the cylinder $D^{2} \times I$, let $X_{n}$ be a set of $n$ distinct input framed points on a diameter $D^{2} \times\{1\}$ and $Y_{n}$ be a set of $n$ distinct output framed points on a diameter $D^{2} \times\{0\}$, it is a well-known result [1] that the relative Homflypt skein module $K\left(D^{2} \times I, X_{n} \amalg Y_{n}\right)$ is isomorphic to the $n$th Hecke algebra $H_{n}$, which is the quotient of the braid group algebra $k\left[B_{n}\right]$ by the Homflypt skein relations. This is the geometric realization of $H_{n}$.

In $H_{n}$, there is a set of quasi-idempotent elements $e_{\lambda} \mathrm{s}$ [6] [1] [3]. After normalization, we denote the corresponding idempotent by $y_{\lambda}$ [3].

\subsection{The Birman-Murakami-Wenzl category and the Birman-} Murakami-Wenzl algebra $K_{n}$. In this section 2.2 and section 2.3, we give a summary of the related work of Beliakova and Blanchet. Details, further references to the origin of some of these ideas, and related results of others can be found in [2]. We also provide some figures to illustrate the ideas. 
Definition 4. The relative Kauffman skein module. Let $X=$ $\left\{x_{1}, x_{2}, \cdots, x_{n}, y_{1}, y_{2}, \cdots, y_{n}\right\}$ be a finite set of $2 n$ framed points in the boundary $\partial M$. The relative skein module $K(M, X)$ is the $k$-module generated by relative framed links in $(M, \partial M)$ such that $L \cap \partial M=$ $\partial L=\left\{x_{i}, y_{i}\right\}$ with the induced framing, considered up to an ambient isotopy fixing $\partial M$, quotient by the Kauffman skein relations.

The Birman-Murakami-Wenzl category $K$ consists of: an object in $K$ is a disc $D^{2}$ equipped with a finite set of points and a nonzero vector at each point. If $\beta=\left(D^{2}, l_{0}\right)$ and $\gamma=\left(D^{2}, l_{1}\right)$ are objects, the module $\operatorname{Hom}_{K}(\beta, \gamma)$ is $K\left(D^{2} \times[0,1], l_{0} \times 0 \amalg l_{1} \times 1\right)$. For a Young diagram $\lambda$, we denote by $\square_{\lambda}$ the object of the category $K$ formed with one point assigned for each cell of $\lambda$. We will use the notation $K(\beta, \gamma)$ for $\operatorname{Hom}_{K}(\beta, \gamma)$. For composition of morphisms $f$ and $g$, it's by stacking $f$ on the top of $g$ :

$$
\begin{gathered}
K(\beta, \gamma) \times K(\gamma, \delta) \rightarrow K(\beta, \delta), \\
(f, g) \rightarrow f g .
\end{gathered}
$$

Note that Beliakova and Blanchet choose to stack the second one on the top of the first. Here we follow the convention as in [5] [1].

As a special case, in the cylinder $D^{2} \times I$, let $X_{n}$ be a set of $n$ distinct framed points on a diameter $D^{2} \times\{1\}$ and $Y_{n}$ be a set of $n$ distinct framed points on a diameter $D^{2} \times\{0\}$, then the relative Kauffman skein module $K\left(D^{2} \times I, X_{n} \amalg Y_{n}\right)$ is isomorphic to the Birman-MurakamiWenzl algebra $K_{n}$, which is the quotient of the braid group algebra $k\left[B_{n}\right]$ by the Kauffman skein relations.

The Birman-Murakami-Wenzl algebra $K_{n}$ is generated by the identity $\mathbf{1}_{n}$, positive transpositions $e_{1}, e_{2}, \cdots, e_{n-1}$ and hooks $h_{1}, h_{2}, \cdots$, $h_{n-1}$ as the following:

$$
\begin{aligned}
& e_{i}=|\cdots| \sum_{h_{i}}^{i}|\cdots| \\
& h_{i}=|\cdots| \bigodot_{i+1}^{i}|\cdots|
\end{aligned}
$$

for $1 \leq i \leq n-1$.

Then $K_{n}$ is the braid group algebra $k\left[B_{n}\right]$ quotient by the following relations:

$$
\begin{aligned}
& \left(B_{1}\right) e_{i} e_{i+1} e_{i}=e_{i+1} e_{i} e_{i+1}, \\
& \left(B_{2}\right) e_{i} e_{j}=e_{j} e_{i},|i-j| \geq 2, \\
& \left(R_{1}\right) h_{i} e_{i}=\alpha^{-1} h_{i}, \\
& \left(R_{2}\right) h_{i} e_{i-1}{ }^{ \pm 1} h_{i}=\alpha^{ \pm 1} h_{i},
\end{aligned}
$$


$(K) e_{i}-e_{i}^{-1}=\left(s-s^{-1}\right)\left(\mathbf{1}_{n}-h_{i}\right)$.

Let $I_{n}$ be the ideal generated by $h_{n-1}$. Note that $I_{n}=\{(a \otimes$ $\left.\left.\mathbf{1}_{1}\right) h_{n-1}\left(b \otimes \mathbf{1}_{1}\right): a, b \in K_{n-1}\right\}$. The quotient of $K_{n}$ by the ideal $I_{n}$ is isomorphic to the $n$th Hecke algebra $H_{n}$. We denote the canonical projection map by $\pi_{n}$ :

$$
\pi_{n}: K_{n} \rightarrow H_{n}
$$

Theorem 1. Over $\mathbb{Q}(\alpha, s)$, the field of rational functions in $\alpha, s$, there exists a multiplicative homomorphism $s_{n}: H_{n} \rightarrow K_{n}$, such that

$$
\begin{gathered}
\pi_{n} \circ s_{n}=i d_{H_{n}}, \\
s_{n}(x) y=y s_{n}(x)=0, \forall x \in H_{n}, \forall y \in I_{n} .
\end{gathered}
$$

Corollary 1. $K_{n} \cong H_{n} \oplus I_{n}$.

Let $\widetilde{y}_{\lambda}=s_{n}\left(y_{\lambda}\right)$.

2.3. A basis for the Birman-Murakami-Wenzl algebra $K_{n}$. We call a sequence $\Lambda=\left(\Lambda_{1}, \cdots, \Lambda_{n}\right)$ of Young diagrams an up and down tableau of length $n$ and shape $\Lambda_{n}$ if two consecutive Young diagrams $\Lambda_{i}$ and $\Lambda_{i+1}$ differ by exactly one cell. We observe that in an up and down tableau $\Lambda=\left(\Lambda_{1}, \cdots, \Lambda_{n}\right)$ of length $n$, the size of $\Lambda_{n}$ is either $n$ or less than $n$ by an even number.

For an up and down tableau $\Lambda$ of length $n$, we denote by $\Lambda^{\prime}$ the tableau of length $n-1$ obtained by removing the last Young diagram in the sequence $\Lambda$. We define $a_{\Lambda} \in K\left(n, \square_{\Lambda}\right)$ and $b_{\Lambda} \in K\left(\square_{\Lambda}, n\right)$ by

$$
a_{1}=b_{1}=\mathbf{1}_{1} .
$$

If $\left|\Lambda_{n}\right|=\left|\Lambda_{n-1}\right|+1$, then

$$
\begin{aligned}
& a_{\Lambda}=\left(a_{\Lambda^{\prime}} \otimes \mathbf{1}_{1}\right) \widetilde{y}_{\Lambda_{n}}, \\
& b_{\Lambda}=\widetilde{y}_{\Lambda_{n}}\left(b_{\Lambda^{\prime}} \otimes \mathbf{1}_{1}\right) ;
\end{aligned}
$$

if $\left|\Lambda_{n}\right|=\left|\Lambda_{n-1}\right|-1$, then

$$
\begin{gathered}
a_{\Lambda}=\frac{<\Lambda_{n}>}{<\Lambda_{n-1}>}\left(a_{\Lambda^{\prime}} \otimes \mathbf{1}_{1}\right)\left(\widetilde{y}_{\Lambda_{n}} \otimes \cup\right), \\
b_{\Lambda}=\left(\widetilde{y}_{\Lambda_{n}} \otimes \cap\right)\left(b_{\Lambda^{\prime}} \otimes \mathbf{1}_{1}\right) .
\end{gathered}
$$

Here $\langle\lambda\rangle$ is the quantum dimension 99 associated with $\lambda$, which is the Kauffman polynomial of $\widehat{\widetilde{y}}_{\lambda}$ in $S^{3}$. Note that $\langle\lambda\rangle$ is invertible in $\mathbb{Q}(\alpha, s)$ [2] [Theorem 7.5].

We provide the following figures to illustrate the ideas. 
(1) If $\left|\Lambda_{n}\right|=\left|\Lambda_{n-1}\right|+1$, then

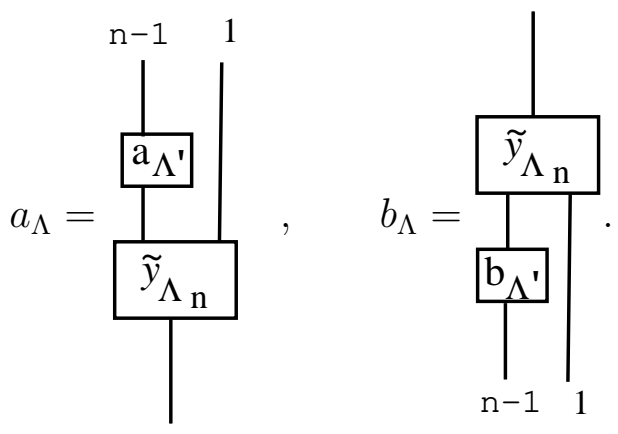

(2) If $\left|\Lambda_{n}\right|=\left|\Lambda_{n-1}\right|-1$, then

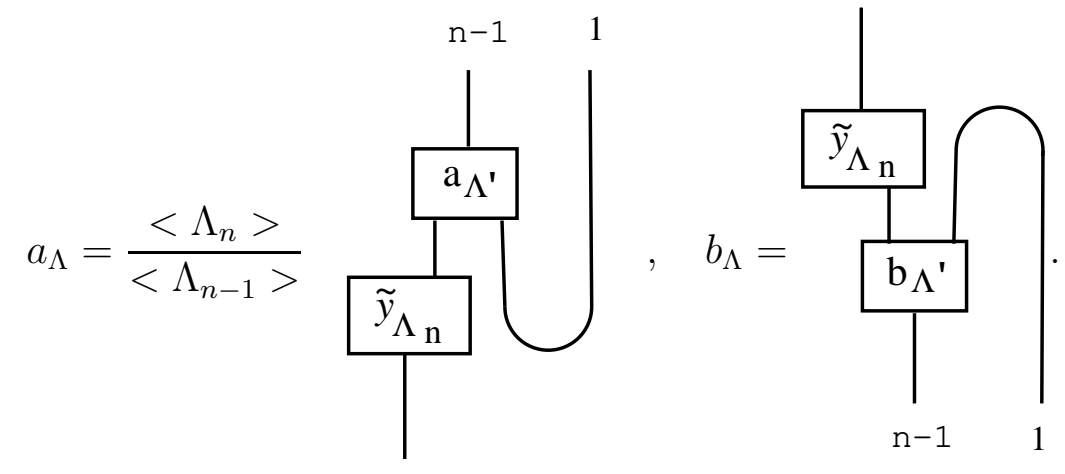

Theorem 2. The family $a_{\Lambda} b_{\Gamma}$ for all up and down tableaux $\Lambda, \Gamma$ of length $n$ such that $\Lambda_{n}=\Gamma_{n}$ forms a basis for $K_{n}$.

(1) If $\left|\Lambda_{n}\right|=\left|\Lambda_{n-1}\right|+1$ (so is $\left|\Gamma_{n}\right|=\left|\Gamma_{n-1}\right|+1$ ), then

$$
a_{\Lambda} b_{\Gamma}=\frac{\tilde{y}_{\Lambda_{\mathrm{n}}}^{\mathrm{a}_{\Lambda^{\prime}}}}{\tilde{\mathrm{b}}_{\Gamma}}
$$


(2) If $\left|\Lambda_{n}\right|=\left|\Lambda_{n-1}\right|-1$ (so is $\left|\Gamma_{n}\right|=\left|\Gamma_{n-1}\right|-1$ ), then

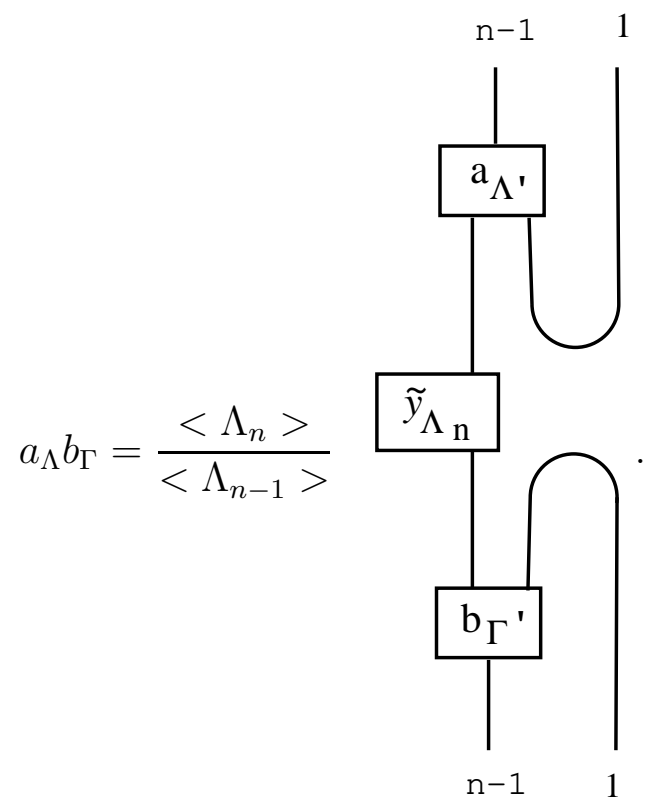

Let $\Lambda=\left(\Lambda_{1}, \cdots, \Lambda_{n}\right), \Gamma=\left(\Gamma_{1}, \cdots, \Gamma_{n}\right)$ be two up and down tableaux of length $n$. If $\Lambda=\Gamma$, i.e. $\Lambda_{i}=\Gamma_{i}$ for $1 \leq i \leq n$, then $b_{\Gamma} a_{\Lambda}=\widetilde{y}_{\Lambda_{n}}$; otherwise $b_{\Lambda} a_{\Gamma}=0$. This follows from the corresponding properties in the Hecke category [3]. These properties will be used in the following section.

2.4. The Kauffman skein module of the solid torus $S^{1} \times D^{2}$. There is a natural wiring of the cylinder $D^{2} \times I$ into the solid torus $S^{1} \times D^{2}$

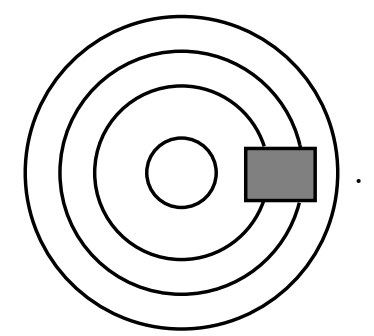

We denote the image of $K_{n}$ under the above wiring by $\widehat{K_{n}}$.

Theorem 3. $\widehat{K_{n}} \subseteq K\left(S^{1} \times D^{2}\right)$ has a basis given by the collection $\left\{\widehat{\widetilde{y}}_{\lambda}:|\lambda|\right.$ is either $n$ or less than $n$ by an even number $\}$.

Proof. By Theorem 2 in the previous section, $K_{n}$ has a basis given by the family $a_{\Lambda} b_{\Gamma}$, so $\widehat{K_{n}}$ is generated by the family $\widehat{a_{\Lambda} b_{\Gamma}}$. Since $\widehat{a_{\Lambda} b_{\Gamma}}=\widehat{b_{\Gamma} a_{\Lambda}}=\delta_{\Lambda \Gamma} \widehat{b_{\Lambda} a_{\Lambda}}=\delta_{\Lambda \Gamma} \widehat{\widetilde{y}}_{\Lambda_{n}}$, where $\Lambda_{n}$ is a Young diagram of size 
either $n$ or less than $n$ by an even number. Thus $\widehat{K_{n}}$ is generated by the collection $\left\{\widehat{\widetilde{y}}_{\lambda}:|\lambda|\right.$ is either $n$ or less than $n$ by an even number $\}$.

Now we want to prove the above generating set is linearly independent. We show this by comparing the dimensions.

From Corollary 1 , we have $K_{n} \cong H_{n} \oplus I_{n}$, so $\widehat{K_{n}} \cong \widehat{H_{n}}+\widehat{I_{n}}$. As $I_{n}=\left\{\left(a \otimes \mathbf{1}_{1}\right) h_{n-1}\left(b \otimes \mathbf{1}_{1}\right): a, b \in K_{n-1}\right\}$, a typical element in $\widehat{I_{n}}$ looks like the following:

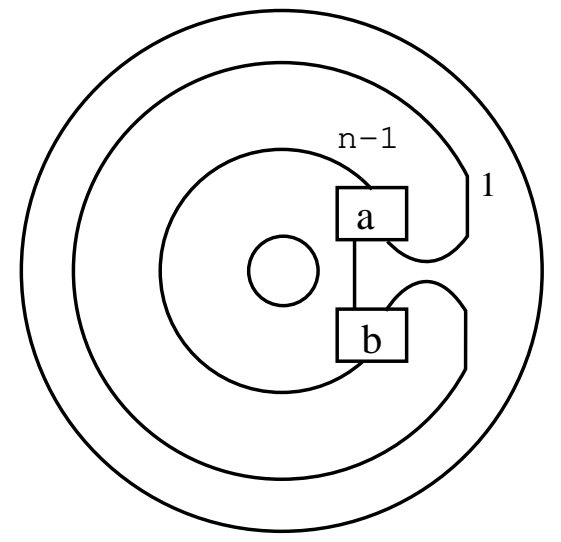

where $a, b \in K_{n-1}$. We can see that the above element is in $\widehat{K_{n-2}}$. On the other hand, we have $\widehat{K_{n-2}} \subseteq \widehat{I_{n}}$, therefore $\widehat{I_{n}}=\widehat{K_{n-2}}$. i.e. $\widehat{K_{n}} \cong \widehat{H_{n}}+\widehat{K_{n-2}}$.

Repeating the process for $\widehat{K_{n-2}}$, we conclude that

$$
\widehat{K_{n}} \cong \begin{cases}\widehat{H_{1}}+\widehat{H_{3}}+\cdots+\widehat{H_{n}}, & \text { if } n \text { is odd; } \\ <\phi>+\widehat{H_{2}}+\widehat{H_{4}}+\cdots+\widehat{H_{n}}, & \text { if } n \text { is even; }\end{cases}
$$

where $\phi$ is the empty link.

Since $\widehat{H}_{i} \cap \widehat{H}_{j}=0$ whenever $i \neq j$ in the Homflypt skein module of the solid torus, the above decomposition is a direct sum.

$$
\widehat{K_{n}} \cong \begin{cases}\widehat{H_{1}} \oplus \widehat{H_{3}} \oplus \cdots \oplus \widehat{H_{n}}, & \text { if } n \text { is odd } \\ <\phi>\oplus \widehat{H_{2}} \oplus \widehat{H_{4}} \oplus \cdots \oplus \widehat{H_{n}}, & \text { if } n \text { is even }\end{cases}
$$

Therefore we have the following equality for the dimensions.

$$
\operatorname{dim}\left(\widehat{K_{n}}\right)= \begin{cases}\operatorname{dim}\left(\widehat{H_{1}}\right)+\operatorname{dim}\left(\widehat{H_{3}}\right)+\cdots+\operatorname{dim}\left(\widehat{H_{n}}\right), & \text { if } n \text { is odd } \\ 1+\operatorname{dim}\left(\widehat{H_{2}}\right)+\operatorname{dim}\left(\widehat{H_{4}}\right)+\cdots+\operatorname{dim}\left(\widehat{H_{n}}\right), & \text { if } n \text { is even }\end{cases}
$$

As we know from [1] that the dimension of $\widehat{H}_{i}\left(=C_{i}\right)$ in $S\left(S^{1} \times D^{2}\right)$ is equal to the number of Young diagrams of size $i$. By comparing the 
number of generators in the set $\left\{\widehat{\widetilde{y}}_{\lambda}:|\lambda|\right.$ is either $n$ or less than $n$ by an even number $\}$ and the dimension of $\widehat{K_{n}}$, they are equal, so the given generating set must be linearly independent. Thus it forms a basis for $\widehat{K_{n}}$.

Corollary 2. The collection of all the elements $\left\{\widehat{\widetilde{y}}_{\lambda}: \lambda\right.$ is any Young diagram $\}$ forms a basis for $K\left(S^{1} \times D^{2}\right)$ over $\mathbb{Q}(\alpha, s)$, where

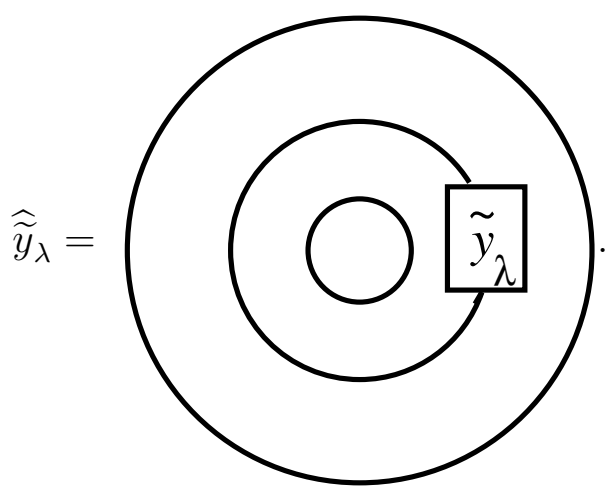

Proof. Let $L$ be a framed link in $S^{1} \times D^{2}$, up to a scalar multiple, then $L$ is the closure of an $n$-strand braid for some integer $n \geq 0$ \& 86.5 Alexander's Braiding Theorem]; the braid quotient by the Kauffman skein relations is in $K_{n}$. Therefore $L \in \widehat{K_{n}}$ in $K\left(S^{1} \times D^{2}\right)$. This shows any element in $K\left(S^{1} \times D^{2}\right)$ lies in $\bigcup_{n \geq 0}{\widehat{K_{n}}}_{n}$, i.e. $K\left(S^{1} \times D^{2}\right) \subseteq \bigcup_{n \geq 0} \widehat{K_{n}}$, hence $K\left(S^{1} \times D^{2}\right)=\bigcup_{n \geq 0} \widehat{K_{n}}$. So we need only to show that the set $\left\{\widehat{\widetilde{y}}_{\lambda}: \lambda\right.$ is a Young diagram $\}$ forms a basis for $\bigcup_{n \geq 0} \widehat{K_{n}}$.

From the previous Theorem, $\widehat{K_{n}} \subseteq K\left(S^{1} \times D^{2}\right)$ has a basis given by the collection $\left\{\widehat{\widetilde{y}}_{\lambda}:|\lambda|\right.$ is either $n$ or less than $n$ by an even number $\}$. It follows that the set $\left\{\widehat{\widetilde{y}}_{\lambda}: 0 \leq|\lambda| \leq n\right\}$ forms a basis for $\bigcup_{0 \leq i \leq n} \widehat{K}_{i}$. As we have $K\left(S^{1} \times D^{2}\right)=\bigcup_{n \geq 0} \widehat{K_{n}}$. The corollary follows by induction on $m$ for $\bigcup_{0 \leq n \leq m} \widehat{K_{n}}$.

2.5. A linear map on $K\left(S^{1} \times D^{2}\right)$. We define a natural linear map $\varphi$ on $K\left(S^{1} \times D^{2}\right)$ in the following:

$$
\varphi: K\left(S^{1} \times D^{2}\right) \rightarrow K\left(S^{1} \times D^{2}\right)
$$




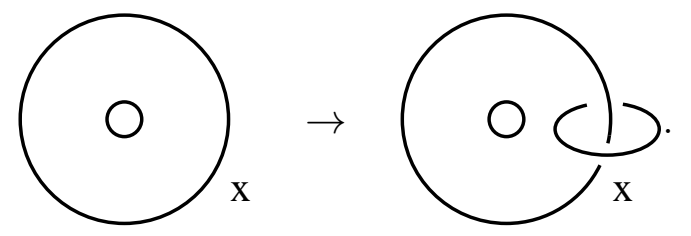

When $X=A_{1}$, where $A_{1}$ is the unknot in the annulus representing the longitude, then $H(n, m)=\varphi^{m}\left(A_{1}\right)$.

Let $\lambda$ be a Young diagram, $\lambda$ has $|\lambda|$ cells indexed by $\{(i, j)\}$. If $c$ is the cell of index $(i, j)$ in $\lambda$, its content $c n(c)$ is defined by $c n(c)=j-i$. We define,

$$
c_{\lambda}=\delta+\left(s-s^{-1}\right)\left(\alpha \sum_{c \in \lambda} s^{2 c n(c)}-\alpha^{-1} \sum_{c \in \lambda} s^{-2 c n(c)}\right)
$$

Note that the scalars $c_{\lambda}$ are different for each Young diagram $\lambda$.

\section{Proposition 2.1.}

$$
\varphi\left(\widehat{\widetilde{y}}_{\lambda}\right)=c_{\lambda} \widehat{\widetilde{y}}_{\lambda}
$$

The proof follows from the following lemma.

\section{Lemma 2.2.}

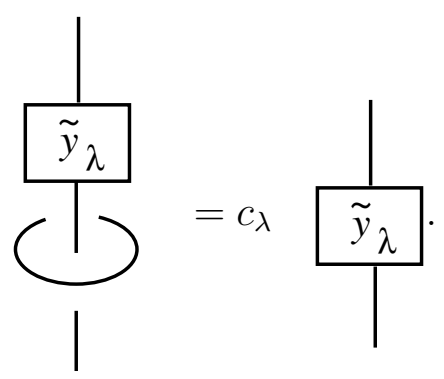

Proof. We proceed by induction on $|\lambda|$.

(1) It's easy to check the result is true when $|\lambda|=0$ and $|\lambda|=1$.

(2) Suppose it is true for $|\lambda|<n$. Now assume $\lambda$ is a Young diagram of size $n$.

First we have the following identity by the absorbing property given in [2][Chapter 5]:

$$
\widetilde{y}_{\lambda}=\widetilde{y}_{\lambda}\left(\widetilde{y}_{\lambda^{\prime}} \otimes 1\right) \widetilde{y}_{\lambda}
$$




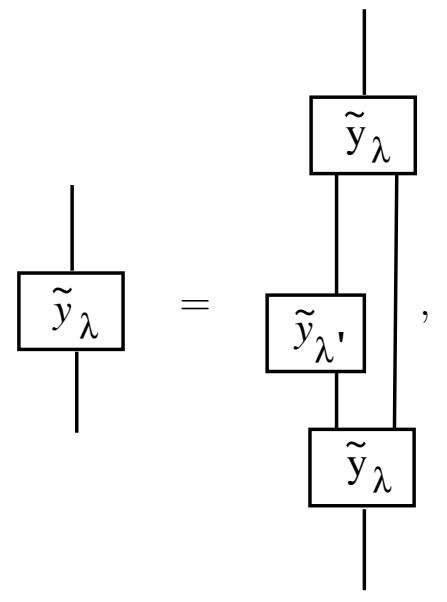

and the skein relation [2, Prop. 6.1]:

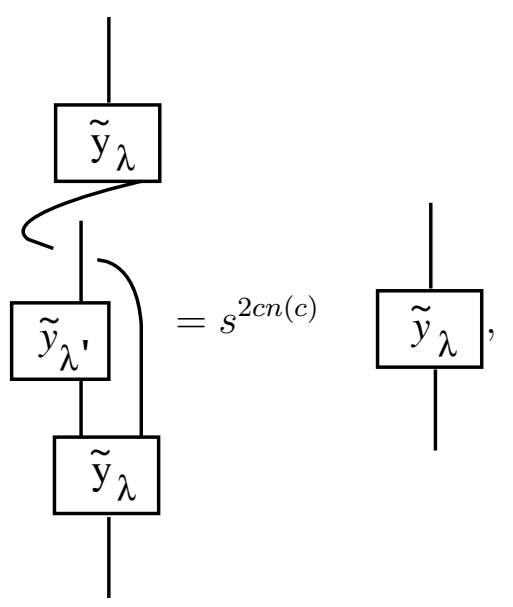

and from the above skein relation, we can develop the following relation,

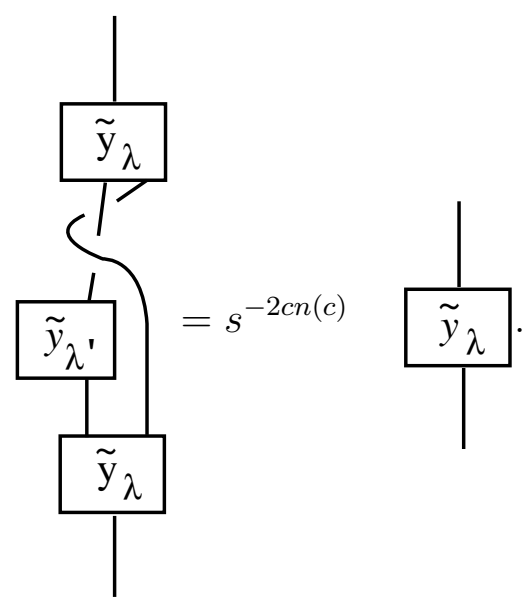

where $c n(c)$ is the content of the extreme cell $c$ of $\lambda$ to be removed to obtain $\lambda^{\prime}$. 
Now, apply the Kauffman skein relation to the following,

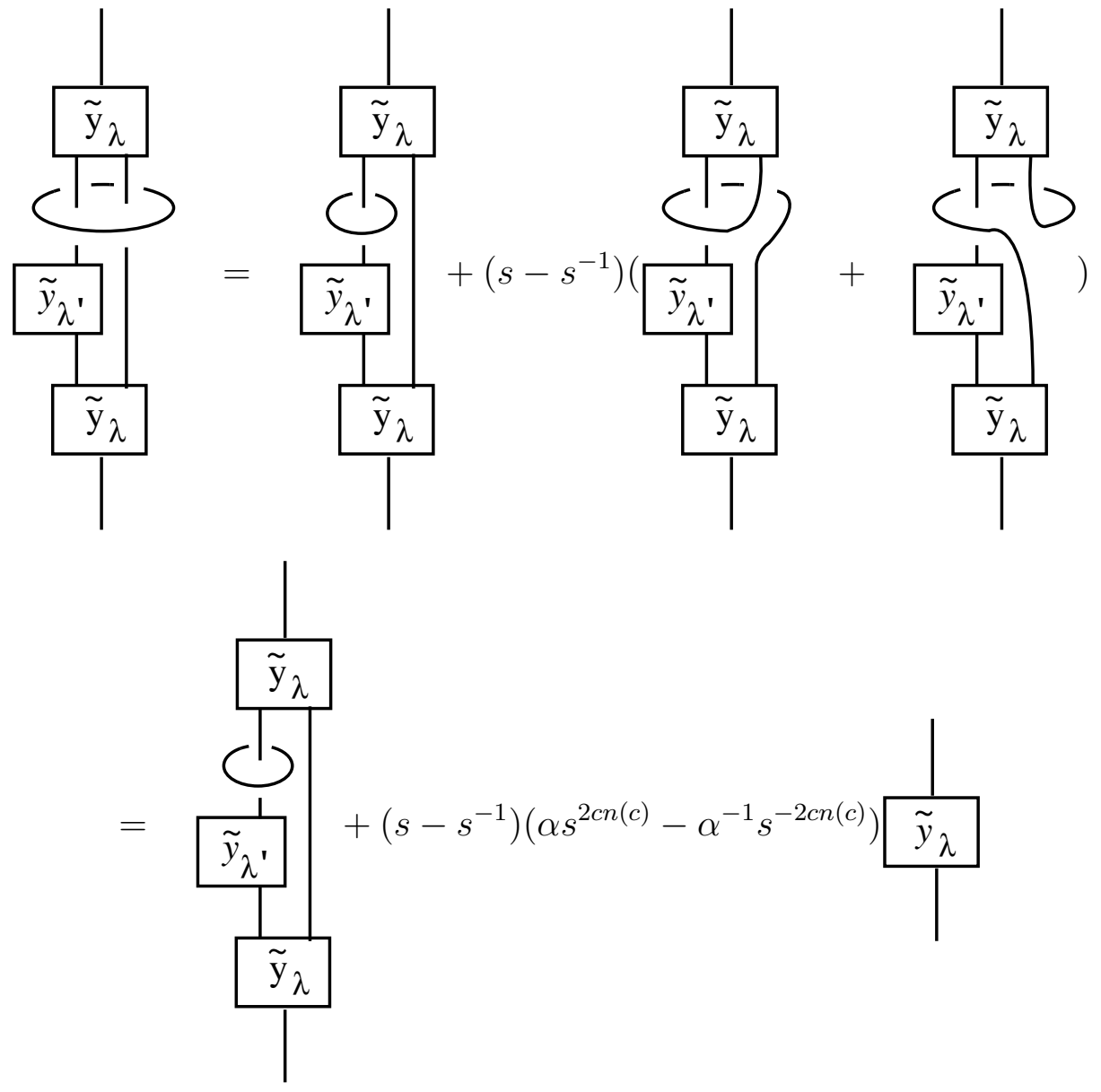

(by induction on $\lambda^{\prime}$ )

$$
\begin{aligned}
& =c_{\lambda^{\prime}} \\
& =c_{\lambda} \widetilde{y}_{\lambda} .
\end{aligned}
$$


Corollary 3. For $m \in \mathbf{Z}^{+}$,

$$
\varphi^{m}\left(\widehat{\widetilde{y}}_{\lambda}\right)=\left(c_{\lambda}\right)^{m} \widehat{\widetilde{y}}_{\lambda}
$$

Theorem 4. The eigenvalues of $\left.\varphi\right|_{\widehat{K_{n}}}$ are all distinct.

Proof. Let $\lambda$ be a Young diagram of size $i$. From above, we have $\varphi\left(\widehat{\widetilde{y}}_{\lambda}\right)=\left(c_{\lambda}\right) \widehat{\widetilde{y}}_{\lambda}$, so $\widehat{\widetilde{y}}_{\lambda}$ is an eigenvector of $\varphi$ with eigenvalue $c_{\lambda}$. There are $p(i)$ of these eigenvectors all with distinct eigenvalues, where $p(i)$ is the number of Young diagrams of size $i$. Now the set $\left\{\widehat{\widetilde{y}}_{\lambda}:|\lambda|=n\right.$ or less than $n$ by an even number $\}$ forms a basis for the subspace $\widehat{K_{n}}$, each $\widehat{\widetilde{y}}_{\lambda}$ is an eigenvector with a unique eigenvalue, we conclude that the eigenspaces are all 1-dimensional.

This establishes that any element in $\widehat{K}_{n}$ can be written as a linear combination of the $\widehat{\widetilde{y}}_{\lambda}$ with $|\lambda|$ being equal to $n$ or less than $n$ by an even number. It follows that any element of $\widehat{K_{n}}$ which is an eigenvector of $\varphi$ must be a multiple of some $\widehat{\widetilde{y}}_{\lambda}$ as given above.

\section{Kauffman polynomials of SOME Generalized Hopf Links}

In this section, we will apply the techniques described in the previous sections to show that it is possible to compute the Kauffman polynomial of some generalized Hopf links. We will give specific examples in the next section.

Consider $H(n, 0)$ in $K\left(S^{1} \times D^{2}\right)$, then $H(n, 0)=A_{1}^{n}\left(A_{1}\right.$ denotes the unknot in the annulus representing the longitude).

From the previous section, a basis for $\widehat{K}_{n}$ is given by the set $\left\{\widehat{\widetilde{y}}_{\lambda}\right.$ : $|\lambda|=n$ or less than $n$ by an even number $\}$. We have

$$
A_{1}^{n}=\sum_{\lambda} d_{\lambda} \widehat{\widetilde{y}}_{\lambda}
$$

where the sum is over all Young diagram $\lambda$ with $|\lambda|$ equal to $n$ or less than $n$ by an even number.

Therefore, in $K\left(S^{1} \times D^{2}\right)$,

$$
H(n, m)=\varphi^{m}\left(A_{1}^{n}\right)=\sum_{|\lambda|=i} d_{\lambda} \varphi^{m}\left(\widehat{\widetilde{y}}_{\lambda}\right)=\sum_{|\lambda|=i} d_{\lambda} c_{\lambda}^{m} \widehat{\widetilde{y}}_{\lambda} .
$$

So by evaluating in the plane, the Kauffman polynomial of $H(n, m)$ is:

$$
<H(n, m)>=\sum_{|\lambda|=i} d_{\lambda} c_{\lambda}^{m}<\lambda>,
$$


where $\langle\lambda\rangle$ is the Kauffman polynomial of $\widehat{\widetilde{y}}_{\lambda}$ given by Wenzl [9] [Theorem 5.5] and by Beliakova and Blanchet [2] [Theorem 7.5].

4. The Kauffman polynomials of $H(1, n)$ and $H(2, n)$

4.1. The Kauffman polynomials of $H(1, n)$. When $\lambda$ is a Young diagram with a single cell, we have $c_{\lambda}=\left[\delta+\left(s-s^{-1}\right)\left(\alpha-\alpha^{-1}\right)\right]$.

$$
<\varphi\left(\widehat{\widetilde{y}}_{\lambda}\right)>=<\text { Hopflink }>=\delta\left[\delta+\left(s-s^{-1}\right)\left(\alpha-\alpha^{-1}\right)\right] .
$$

Therefore,

$$
<H(1, n)>=<\varphi^{n}\left(\widehat{\widetilde{y}}_{\lambda}\right)>=\delta\left[\delta+\left(s-s^{-1}\right)\left(\alpha-\alpha^{-1}\right)\right]^{n} .
$$

4.2. The Kauffman polynomials of $H(2, n)$. Let $\lambda$ be the Young diagram of size two with the two cells on one row, let $\mu$ be the Young diagram of size two with the two cells on one column.

$$
\lambda=\square, \quad \mu=\square \text {. }
$$

Recall in the Hecke algebra $H_{2}$, the idempotents corresponding to $\lambda$ and $\mu$ are the symmetrizer and antisymmetrizer denoted by $f_{2}$ and $g_{2}$ by Beliakova and Blanchet [2] [Chapter 2], where

$$
\left.f_{2}=\frac{s^{-1}}{s+s^{-1}}\right)\left(+\frac{s^{-1}}{s+s^{-1}} \searrow\right.
$$

and

$$
\left.g_{2}=\frac{s}{s+s^{-1}}\right)\left(-\frac{s^{-1}}{s+s^{-1}} \searrow\right. \text { ऽ. }
$$

From the definition of $f_{2}$ and $g_{2}$, in $H_{2}$, we have,

$$
)\left(=f_{2}+g_{2}\right. \text {. }
$$

Now apply the multiplicative homomorphism $s_{2}: H_{2} \rightarrow K_{2}$, from the construction of $s_{2}$ in the proof of [2][Theorem 3.1],

Therefore,

$$
\left.s_{2}()()=\right)\left(-\delta^{-1} \asymp .\right.
$$

$$
\begin{aligned}
& =s_{2}()()+\delta^{-1} \leftrightharpoons \\
& =s_{2}\left(f_{2}\right)+s_{2}\left(g_{2}\right)+\delta^{-1} \leftrightharpoons
\end{aligned}
$$

Now by embedding this into the skein of the annulus, we have, 


$$
\begin{aligned}
H(2,0) & =\widehat{\widetilde{y}}_{\lambda}+\widehat{\widetilde{y}}_{\mu}+\delta^{-1} \delta \phi \\
& =\widehat{\widetilde{y}}_{\lambda}+\widehat{\widetilde{y}}_{\mu}+\phi .
\end{aligned}
$$

So the Kauffman polynomial of $H(2, n)$ is

$$
\begin{aligned}
<H(2, n)> & =<\varphi^{n}\left(\widehat{\widetilde{y}_{\lambda}}+\widehat{\widetilde{y}}_{\mu}+\phi\right)> \\
& =<\varphi^{n}\left(\widehat{\widetilde{y}_{\lambda}}\right)>+<\varphi^{n}\left(\widehat{\widetilde{y}}_{\mu}\right)>+<\varphi^{n}(\phi)> \\
& =c_{\lambda}^{n}<\lambda>+c_{\mu}^{n}<\mu>+\delta^{n},
\end{aligned}
$$

where $c_{\lambda}=\delta+\left(s-s^{-1}\right)\left(\alpha\left(1+s^{2}\right)-\alpha^{-1}\left(1+s^{-2}\right)\right)$ and $c_{\mu}=\delta+(s-$ $\left.s^{-1}\right)\left(\alpha\left(1+s^{-2}\right)-\alpha^{-1}\left(1+s^{2}\right)\right)$, and from [2] [Theorem 7.5],

$$
<\lambda>=\frac{\left(\alpha-\alpha^{-1}\right)\left(\alpha s-\alpha^{-1} s^{-1}+s^{2}-s^{-2}\right)}{\left(s+s^{-1}\right)\left(\left(s-s^{-1}\right)^{2}\right.}
$$

and

$$
<\mu>=\frac{\left(\alpha-\alpha^{-1}\right)\left(\alpha s^{-1}-\alpha^{-1} s+s^{2}-s^{-2}\right)}{\left(s+s^{-1}\right)\left(\left(s-s^{-1}\right)^{2}\right.} .
$$

As a special case, when $n=0$, we have $\langle H(2,0)\rangle=\langle\lambda\rangle+\langle\mu\rangle$ $+1=\delta^{2}$.

\section{REFERENCES}

[1] A. K. Aiston and H. R. Morton, Idempotents of Hecke algebras of type A, J. of Knot Theory and Ram. 7 No 4 (1998), 463-487.

[2] A. Beliakava and C. Blanchet, Skein construction of Idempotents in BirmanMurakami-Wenzl algebras, Preprint, ArXiv: math. QA/000614.

[3] C. Blanchet, Hecke algebras, modular categories and 3-manifolds quantum invariants, Topology (39) 1 (2000), 193-223.

[4] T.-H. Chan, Homfly polynomial of some generalized Hopf links, J. Knot Theory Ramif., 9(7):865-883,2000.

[5] P. Gilmer and J. K. Zhong, The Homflypt skein module of $S^{1} \times S^{2}$, Math Zeit., vol 237,pp 769-814 (2001).

[6] A. Gyoja, A q-analogue of Young symmetrizers, Osaka J. Math., 23:841-852, 1986.

[7] H. R. Morton and R. J. Hadji, Homfly polynomials of generalized Hopf links, Preprint, ArXive: math.GT/0106207.

[8] V. V. Prasolov and A. B. Sossinsky, Knots, Links, Braids and 3-Manifolds, American Mathematical Society, QA612.14.P73, 1997.

[9] H. Wenzl, On the structure of Brauer's centralizer algebras, Annals of Math. Vol. 128, 173-193, 1988. 
Department of Mathematics \& Statistics, Louisiana Tech UniverSITY, Ruston, LA 71272

E-mail address: kzhong@coes.latech.edu

Department of Mathematics, The University of Arizona, Tucson, AZ 85721

E-mail address: binlu@math.arizona.edu 